Supporting Information (SI)

\title{
Closed-Cell, Phase Change Material-Encapsulated Monoliths from a Reactive Surfactant-Stabilized High Internal Phase Emulsion for Thermal Energy Storage
}

Tao Zhang, ${ }^{\mathrm{a},}$, Zhiguang $\mathrm{Xu}^{\mathrm{b}}$, Huanjie $\mathrm{Chi}^{\mathrm{a}}$, and Yan Zhao ${ }^{\mathrm{a},{ }^{*}}$

a College of Textile and Clothing Engineering, Soochow University, Suzhou 215123, China.

b China-Australia Institute for Advanced Materials and Manufacturing, Jiaxing University, Jiaxing 314001, China.

Corresponding Authors

E-mail: zhang.tao@suda.edu.cn (T. Zhang); yanzhao@suda.edu.cn (Y. Zhao) 


\section{EXPERIMENTAL SECTION}

Table S1. FAM26- $Y$ recipes

$\begin{array}{lll}\text { FAM26-75 } & \text { FAM26-80 } & \text { FAM26-83 }\end{array}$

Continuous aqueous phase (wt \%)

\begin{tabular}{lccc} 
F127-DA & 1.25 & 1.00 & 0.85 \\
AAm & 5.00 & 4.00 & 3.40 \\
MBAAm & 0.25 & 0.20 & 0.17 \\
$\mathrm{H}_{2} \mathrm{O}$ & 18.50 & 14.80 & 12.58 \\
Total & 25.00 & 20.00 & 17.00 \\
\hline Dispersed oil phase (v/v \%) & & & \\
Octodecane & 74.50 & 79.60 & 82.66 \\
Darocur 1173 & 0.50 & 0.40 & 0.34
\end{tabular}

F127-DA in monomers

$(\%)$

$\begin{array}{lll}26.32 & 26.32 & 26.32\end{array}$

Octodecane fraction in the FAM26- $Y$ (wt \%)

89.9

92.3

93.6 


\section{RESULTS AND DISCUSSION}

\subsection{Chemical structures}

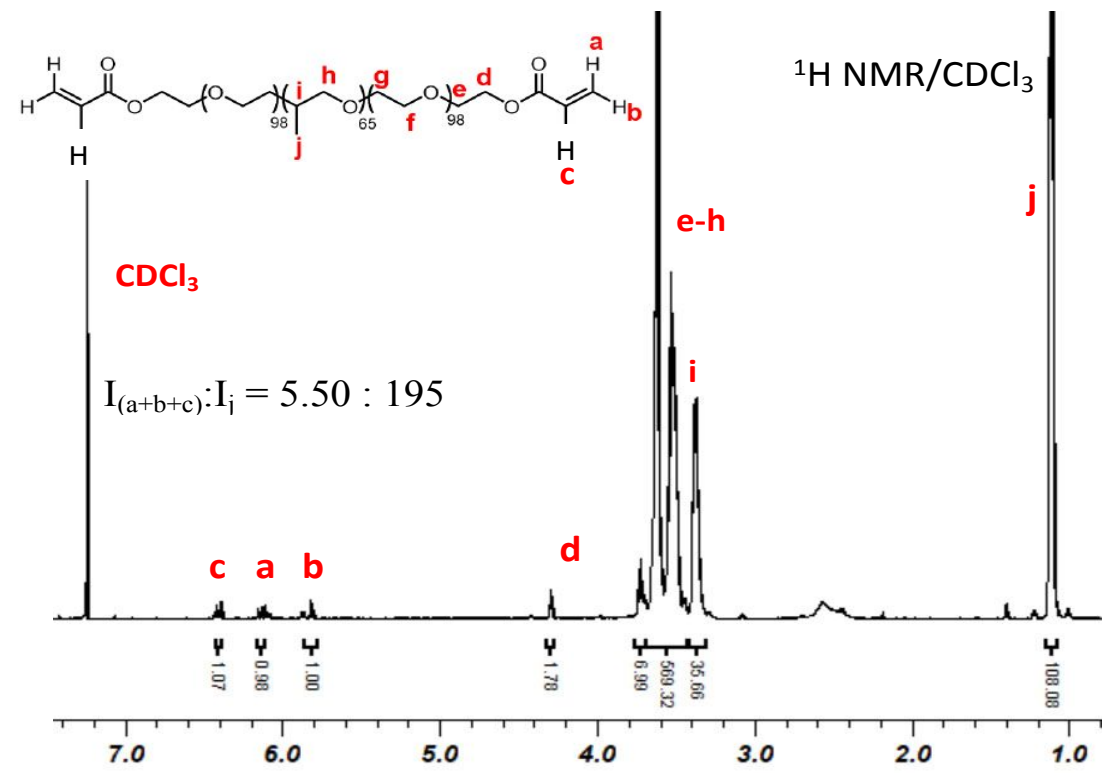

Figure S1. ${ }^{1} \mathrm{H}$ NMR spectrum of F-127-DA in $\mathrm{CDCl}_{3}$.

The ${ }^{1} \mathrm{H}$ NMR spectrum of F-127-DA in Fig. S1 exhibits an integral ratio of 5.5:195 for $I_{(a+b+c)}$ (the $H$ of acrylate) to $I_{j}$ (the methyl of PPO). The theoretical $I_{(a+b+c)}:$ Ij from a complete reaction of F-127 hydroxyl end groups with acryloyl chloride is $6: 195$, the ${ }^{1} \mathrm{H}$ NMR results showed that about $92 \%$ of the hydroxyl end groups of F127 were end-capped successfully.

\subsection{Porous structure}

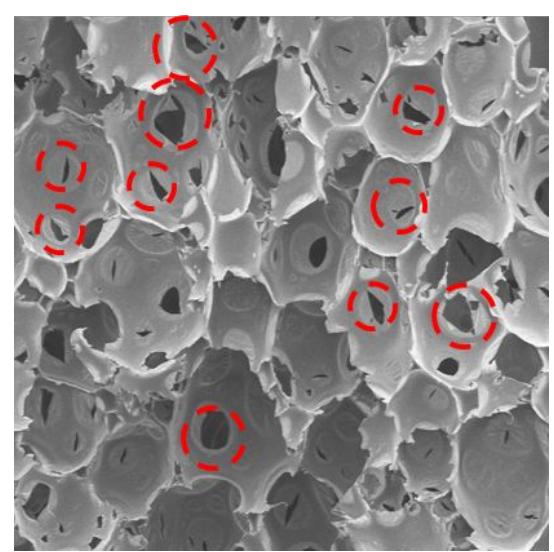

Figure S2. Porous structure (SEM) of FAM19 from HIPEs using hexane as the dispersed phase. The red circles showed the typical interconnecting holes with rapture 
films.

2.3 Void diameter distributions
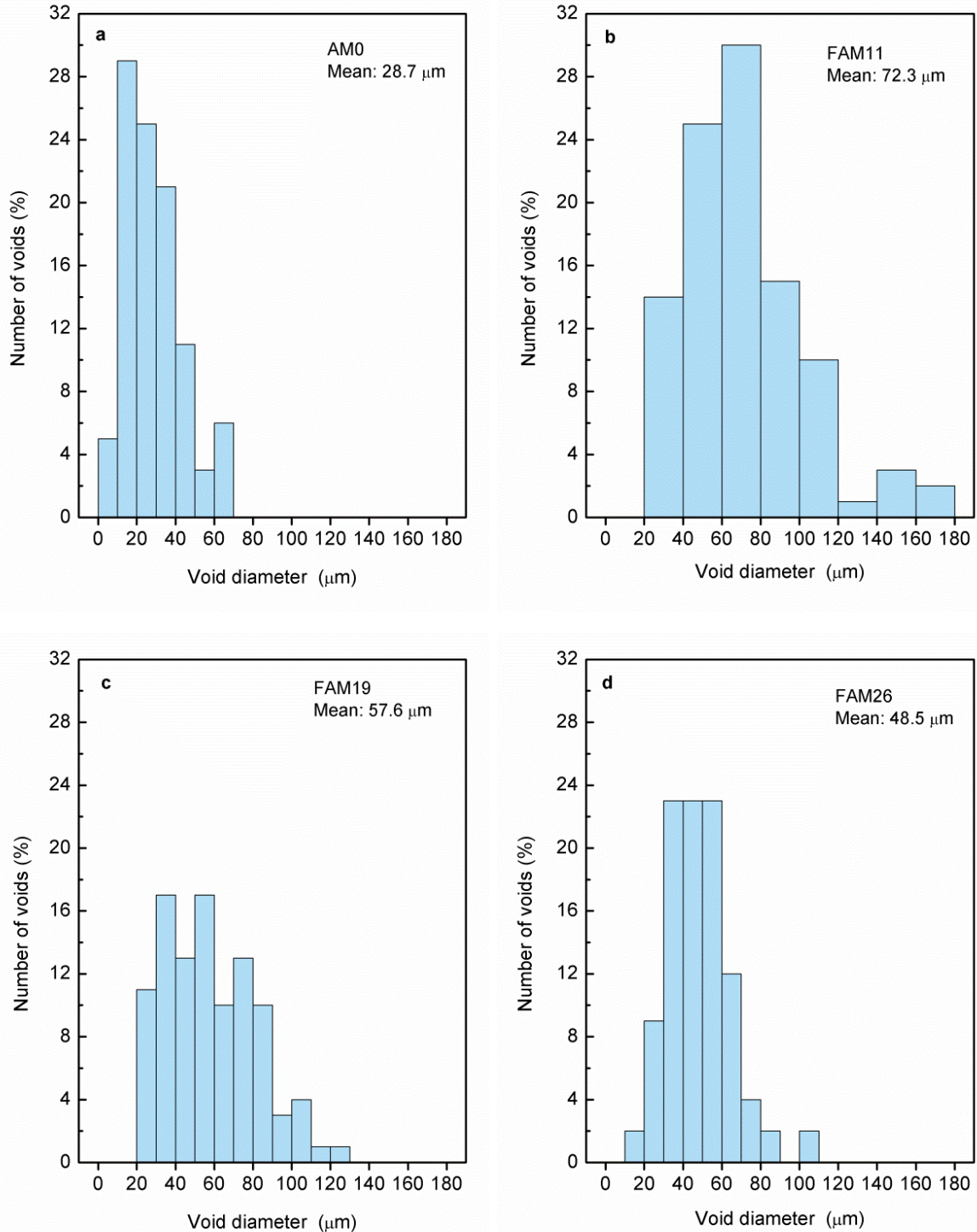

Figure S3. The measured AM0 and FAM $X$ void diameter distributions (as determined from the SEM micrographs): (a) AM0; (b) FAM11; (c) FAM19; (d) FAM26. 